\title{
Suitability of time domain reflectometry (TDR) for moisture content monitoring in historic building walls
}

\author{
Teresa Stingl Freitas ${ }^{1, *}$, Ana Sofia Guimarães ${ }^{1}$, and Vasco Peixoto de Freitas ${ }^{1}$ \\ ${ }^{1}$ Construct - LFC, Faculty of Engineering University of Porto (FEUP), Department of Civil \\ Engineering, Portugal
}

\begin{abstract}
Measuring moisture content in building materials is crucial for the correct diagnosis of buildings pathologies, for the adoption of appropriate intervention measures and for the efficiency evaluation of the treatment solutions applied. There are several different techniques available to measure and monitoring the moisture content in building materials. However, it still remains a great challenge to perform those studies in historical buildings, since minor-destructive techniques are required. Furthermore, if continuous moisture content readings in space are desired, in order to study the moisture transfer phenomenon along the wall thickness, the challenge is even greater. The TDR technique is a relatively new method for measurement of moisture content in building materials with a set of unexplored potentialities capable of satisfying the two mentioned requirements. In this paper, the suitability of the TDR technique to obtain continuous cross-section moisture content profiles has started to be tested on two limestone prototype walls. Each wall was equipped with four TDR probes, designed practically with the same length of the wall thickness and placed at different heights. All the necessary equipment, installation steps, and difficulties are here presented. The preliminary results suggest that the TDR technique is suitable for moisture content monitoring in consolidated porous building materials.
\end{abstract}

\section{Introduction}

The TDR - Time Domain Reflectometry- technique is considered a relatively new method concerning its application to moisture content measurements in construction building materials. This technique was first used to measure dielectric properties of liquid mediums, as the contact between the probe and the medium was easy to guarantee. Since 1980, it suffers a quick development regarding its application for water content measurements in soils. Ten years later, it was already a widely and accepted technique, used in several scientific laboratories to measure dielectric properties of liquids and loose granular soft materials [1]. However, the development of the first experimental configurations suitable for the application of the technique in solid and compact materials only began to appear

*Corresponding author: tsf@fe.up.pt 
later. Indeed, its study and use to measure moisture content in construction materials has only started since 2000 [2]. Nowadays, the knowledge of the TDR technique as a measurement technology is quite advanced and well-founded, but the potentialities and the developments with regard to its application in building materials is a process far from being complete. The difficulty to ensure a good contact between the TDR probe and the construction material under study, as well as the lack of suitable conversion empirical functions capable of providing the moisture content values from the direct results of the TDR measurements are additional obstacles for the application of the technique in building materials. In fact, the quality of the holes drilled to accommodate the waveguides can strongly influence the results if any air layer is present between the TDR probe and the material under test. Drilling good quality holes can be very challenging and extremely difficult in harder and less workable materials, as happens in the case of historical building walls. In addition, many of the theoretical relationships between the moisture content and the relative dielectric permittivity, $\varepsilon_{\mathrm{r}}$, have arisen for the application of TDR to soil moisture measurements and so they are unsuitable to construction materials. The existing calibration function for soils have shown to consistently overestimate the moisture content when applied to building materials [3]. Despite all these difficulties, the TDR technique has a great potential and a huge applicability for different scenarios, according to the type of TDR probe adopted: multiple rod probes, surface TDR probes and wire-like sensing elements. In the last years, many studies were developed about the use of the TDR technique for measuring moisture content in construction building materials, with some results for: cellular concrete [3,4], sandstone [3], volcanic tuff walls [2] and red ceramic brick [5].

\section{The TDR - Time Domain Reflectometry - technique}

\subsection{Basic principles}

Typically, in TDR measurements an electromagnetic (EM) signal is propagated along a probe inserted into the material under test. Any impedance variations causes the partial reflection of the propagating signal. The reflected signal carries information on the dielectric characteristics of the material under study and is acquired by the TDR unit. Through a suitable data processing program, the reflection coefficient, $\rho$ - ratio between the amplitude of the reflected signal and the amplitude of the generated signal - is displayed as a function of the apparent distance $\left(\mathrm{d}_{\mathrm{app}}\right)$ in a graphic called reflectogram. The quantity $\mathrm{d}_{\text {app }}$ is not a real physical distance and can be considered as an "equivalent physical distance" that would be travelled by the EM signal in the same interval of time, if the signal were propagating at the speed of light:

$$
d_{a p p}=\frac{c . t}{2}
$$

Where $\mathrm{c} \approx 3 \times 10^{8} \mathrm{~m} / \mathrm{s}$ is the velocity of light in vacuum and $t$ is the travel time [6]. Figure 1 shows an example of a TDR reflectogram obtained for a $18,5 \mathrm{~cm}$ long two-rod probe inserted in a dry limestone sample. The first derivative of the TDR reflectogram is used to easily identified impedance variations and in this way detect the portion of the reflectogram corresponding to the beginning and end of the probe. After removing the apparent length introduced by the probe-head section, achieved at the end of several measurements performed in mediums with known relative dielectric permittivity, the apparent length, $d_{\text {app}}$, corresponding to the actual physical length of the probe, $d$, can be directly removed from the refletogram. Therefore, the apparent relative dielectric permittivity, $\varepsilon_{\mathrm{app}}$, of any material 
under study can be easily calculated by:

$$
\varepsilon_{a p p}=\left(\frac{d_{a p p}}{d}\right)^{2}
$$

The reflectogram presented in Figure 1 was obtained for a dry limestone sample and for that reason, the reflection coefficient, $\rho$, shows an almost constant value in the portion of path corresponding to the probe's length. Indeed, the behaviour of $\rho$ is strictly associated with the impedance variations encountered by the propagating EM signal. A constant value of $\rho$ means that the dielectric characteristics of the medium are practically uniform. In opposition, variations of $\rho$ indicate that the dielectric characteristics change along the travel electrical path.

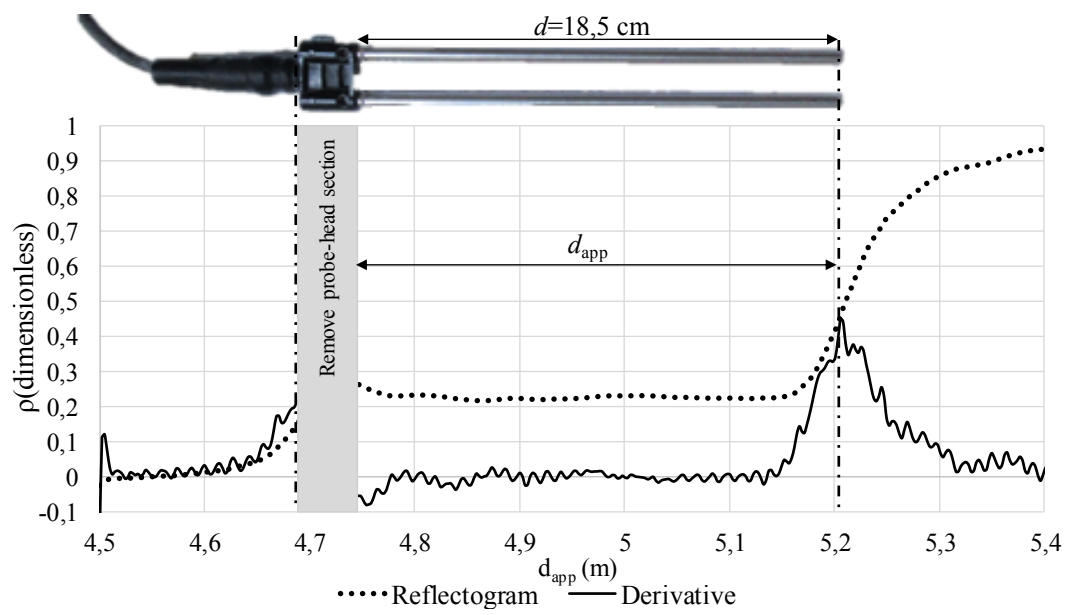

Fig. 1. Example of a TDR reflectogram and corresponding first derivative for a $18,5 \mathrm{~cm}$ long two-rod probe inserted in a dry limestone sample. The peaks of the derivative, represented by the two vertical dashed lines, indicate the most significant impedance changes.

Changes in the moisture content of the study material affect the travel time of the EM signal, leading to changes in the apparent relative dielectric permittivity, $\varepsilon_{\text {app }}$, permitting an indirect determination of the material's water content. Indeed, the presence of water is responsible for the increased propagation time of the EM signal along the probe, and consequently for a bigger value of the apparent relative dielectric permittivity obtained. Despite the temperature dependence of the relative dielectric permittivity of water $\left(\varepsilon_{\mathrm{wat}} \approx 82\right.$ 78 ), its value is significantly higher than both the relative dielectric permittivity of the dry building materials that typically compose the walls $\left(\varepsilon_{\text {dry building materials }} \approx 6-7\right)$ and the air $\left(\varepsilon_{\text {air }} \approx 1\right)$. Therefore, any presence of water is responsible for variations in the dielectric characteristics of the material in the portion that is wet [4]. Figure 2 shows what happens to the reflectograms due to the presence of water. Whenever the moisture content increases in the monitored material, the greater is the apparent distance $\left(d_{a p p}\right)$ and the lower is the reflection coefficient $(\rho)$. Indeed, the quantity $d_{\text {app }}$ obtained in the air is smaller than the one obtained in the dry limestone sample, which in turn is smaller than the one acquired when the probe is placed in water $\left(d_{a p p}\right.$, air $\left.<d_{\text {app, limestone }}<d_{\text {app,water }}\right)$. On the other hand, the reflection coefficient, $\rho$, obtained for the air is higher than the achieved for the dry limestone, which is higher than the recorded in the water $(\rho$, air $>\rho$, limestone $>\rho$, water $)$. 


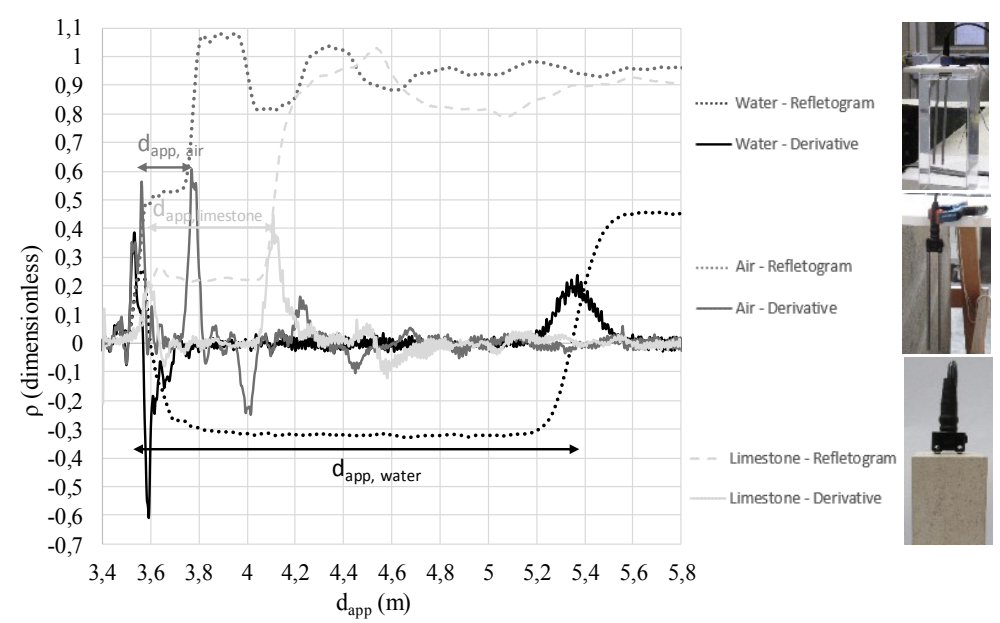

Fig. 2. Example of TDR reflectograms obtained with a $18,5 \mathrm{~cm}$ long two-rod probe placed in water, air and inserted in a dry limestone sample.

\subsection{Equipment}

The TDR measurements were performed through a TDR200 reflectometer. The TDR 200 unit generates a short rise time electromagnetic pulse that is applied to the probes inserted into the material under study. To measure multiple sensors, the TDR 200 requires one or more multiplexers. The role of the multiplexer is to distribute the electromagnetic pulse generated by the TDR200 unit by the different probes in use, allowing that a single electromagnetic pulse can travel across several probes providing multiple measurements. A range of different TDR probes are available: multiple rod probes, surface TDR probes and wire-like sensing elements. In this work, and taking into account that in a near future the purpose is to know the moisture content values at several points along the wall thickness, the multiple rod probes were selected. The probes are composed by two rods, each one with a diameter of $6 \mathrm{~mm}$ and 18,5 cm length. The external spacing between the two rods is 19 $\mathrm{mm}$. Figure 3 shows all the equipment used for the measurements in the two prototype limestone walls, including a detailed picture of the two-rod TDR probes used. To analyse the reflected signal that comes from the TDR probes an appropriate Labview-based software was used. The direct output of the software is a reflectogram.



Fig. 3. Equipment used for the measurements: TDR200, multiplexer ( 8 channels), two-rod TDR probes and coaxial connection cables.

\section{Case study}

Two limestone prototype walls (a typical material in Portuguese historical buildings) placed in an indoor environment were used to start studying the suitability of the TDR technique for moisture content measurements in consolidate porous building materials. The test walls 
(Figure 4 (a)) are located inside large tanks, in order to simulate in the future a capillary absorption test. One of the major obstacles pointed out by several authors to the use of the TDR technique in this kind of hard materials is the difficulty of ensuring a good contact between the waveguides and the material under test. To overcome this difficulty, which is at this stage the main goal of this work, a steel auxiliary guide was developed (Figure 4 (b)). In this way, to insert the TDR probes into the wall the steel guide was fixed to the surface of the wall during the drilling to assure parallel and appropriate spaces between the holes. Indeed, the presence of any air layer next to the waveguide will cause the TDR to measure both the air and the limestone when taking a measurement. The lack of consistency between the quality of the holes drilled to accommodate the rods can therefore lead to variations in the moisture content measurements. The slight dimensions of the waveguides allow their introduction into the material with almost any significant interference in the material's porous structure. The method is only little invasive and thus suitable for monitoring in historical building walls. Figure 4 (c) shows the final experimental configuration. Each wall was equipped with four TDR probes placed at different heights.

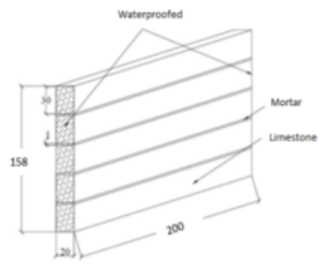

(a)

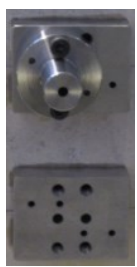

(b)

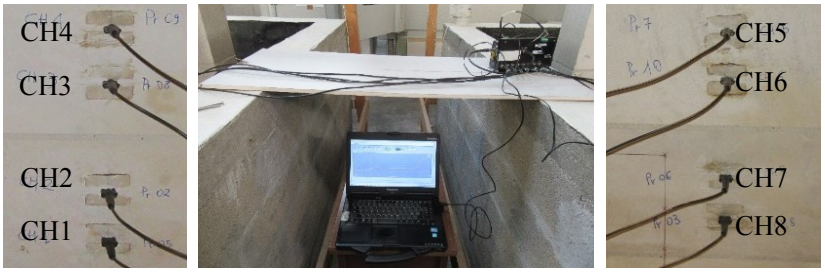

(c)

Fig. 4. (a) Dimensions of the limestone prototype walls used in the experiment; (b) steel guide fixed to the surface of the wall during drilling to ensure good parallel holes; (c) Final experimental setup.

\section{Results and discussion}

The reflectograms and the corresponding first derivatives obtained for the eight TDR probes installed in the two limestone walls are represented in Figure 5. The part of the reflectogram selected corresponds to the distance travelled by the EM signal along the length of the probes, defined by the peaks of the derivative. Despite these preliminary results have been collected with the walls dry, they are really important to perceive the quality of the holes performed and to appreciate the contact between the rods and the limestone reached. As expected, the reflection coefficient, $\rho$, shows an almost constant value for all the eight probes installed, since no water is present capable of leading to significant impedance changes. Besides that, the acquired constant $\rho$ values are also only possible to obtain because no air gap is present between the waveguides and the limestone. The reflection coefficient only shows more variability near the first peak of the derivative due to the impedance changes produced by the probe-head section, that will be removed after a preliminary calibration process. Therefore, these results demonstrate the successful installation of the probes. 


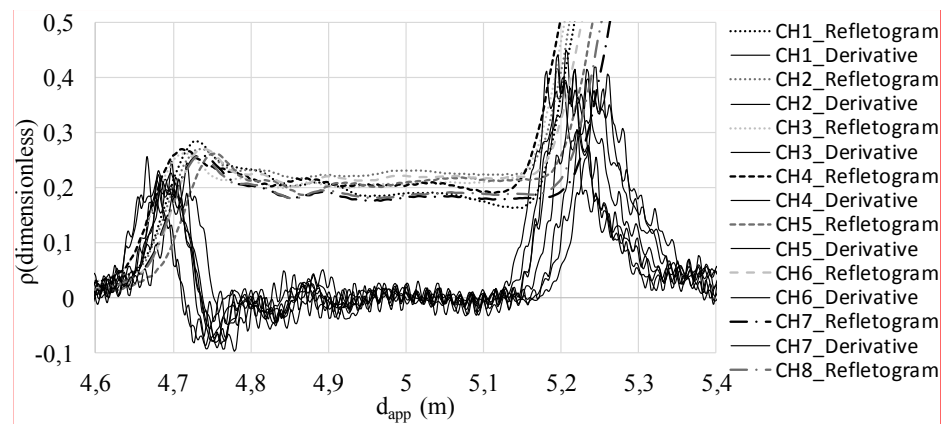

Fig. 5. Reflectograms and corresponding first derivatives for the eight TDR probes installed in the two limestone walls.

\section{Conclusions}

Despite the wide use of the TDR technique, especially for moisture content measurements in soils, the state of the art is rather lacking in TDR applications for moisture content measurements in construction building materials. The difficulty in ensuring a good contact between the TDR probe and the monitored material is pointed out by many authors as one of the major obstacles to the applicability of the technique in consolidated porous building materials. To overcome this difficulty, a steel auxiliary guide was developed to assure parallel and appropriate spaces between the holes. The preliminary results obtain for two limestone prototype walls revealed that no air layer is present next to the waveguides that could lead to variations in moisture content measurements (Figure 5). In the near future, a relationship between the reflection coefficient and the moisture content will be established. In this way, in opposition to the traditional local point sensors, each probe will provide several moisture content readings along the wall thickness (cross section moisture content profiles). The preliminary results obtained and the ability revealed by the technique do detect moisture content variations (Figure 2) suggest its suitability to continuous moisture content monitoring, in a minor-destructive way, in consolidated porous building materials.

Teresa Stingl Freitas would like to thank FCT for financial support through the grant SFRH/BD/121549/2016. This work was financially supported by: UID/ECI/04708/2019- CONSTRUCT Instituto de I\&D em Estruturas e Construções funded by national funds through the FCT/MCTES (PIDDAC). A special acknowledgement to Professor Andrea Cataldo, Dr. Egidio de Benedetto and Giuseppe Cannazza from the University of Salento for all the collaboration in the probes development.

\section{References}

1. G.C. Topp, J.L. Davis, A.P. Annan; Electromagnetic determination of soil water content: Measurements in coaxial transmission lines, Water resources research, vol. 16, p. 547-582 (1980)

2. R. Agliata, L. Mollo, R. Greco; Use of TDR to compare rising damp in three tuff walls made with different mortars, Journal of Materials in Civil Engineering, vol. 29 (2017)

3. M.C. Phillipson, et al., Suitability of time domain reflectometry for monitoring moisture in building materials, Building Services Engineering Research and Technology, vol. 29, p. 261-272 (2008).

4. L. Fiala, M. Pavlíková, Z. Pavlík, Application of TDR method for moisture profiles measurement in cellular concrete, Advanced Materials Research, vol. 982, pp. 11-15 (2014)

5. Z. Suchorab, Noninvasive moisture measurement in building materials, Environmental Engineering IV, Proceedings of the 4th Congress of Environmental Engineering, Poland (2012)

6. A. Cataldo, E.D. Benedetto, G. Cannazza, Advances in Reflectometric Sensing for Industrial Applications, Synthesis Lectures on Emerging Engineering Technologies, vol. 2, (2015) 